\title{
Cluster-mining: An approach for determining core structures of metallic nanoparticles from atomic pair distribution function (PDF) data
}

Soham Banerjee ${ }^{a}$, Chia-Hao Liuª, Kirsten M. O. Jensen ${ }^{b}$, Pavol Juhas ${ }^{c}$, Jennifer D. Lee ${ }^{d}$, Marcus Tofanellie, Christopher J. Ackerson ${ }^{\mathrm{e}}$, Christopher B. Murray ${ }^{\mathrm{d}, \mathrm{f}}$, Simon J. L. Billinge $\mathrm{a}^{\mathrm{a}, \mathrm{g}}$

Email Contact: sb3519@columbia.edu

aDepartment of Applied Physics and Applied Mathematics, Columbia University, New York, NY 10027, USA

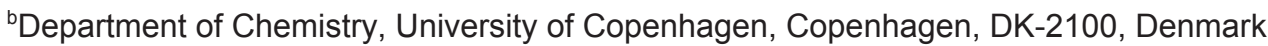

${ }^{\circ}$ Computational Science Initiative, Brookhaven National Laboratory, Upton, NY 11973, USA

dDepartment of Chemistry, University of Pennsylvania, Philadelphia, PA 19104, USA

eDepartment of Chemistry, Colorado State University, Fort Collins, CO, 80523, USA

fDepartment of Materials Science and Engineering, University of Pennsylvania, Philadelphia, PA 19104, USA

${ }^{9}$ Condensed Matter Physics and Materials Science Department, Brookhaven National Laboratory, Upton, New York, 11973, USA

Accurate determination of the structure of metallic nanomaterials is a key step towards understanding and controlling their properties. This is especially challenging for ultrasmall particles where $\mathrm{x}$-ray diffraction data is often not amenable to quantitative crystallographic analysis. In these cases, a better approach is to use PDF analysis. We present a database-driven method for finding and evaluating structural models for these technologically important materials. Rather than fitting a single model with many degrees of freedom, we algorithmically built libraries of nanoparticle clusters from multiple structural motifs. The approach, called cluster-mining, then returns all candidate structure models that are consistent with the data as measured by a goodness of fit. It is highly automated, easy to use, and yields models that are more physically realistic and result in better agreement to the data than models based on cubic close-packed crystallographic cores, often reported in the literature for metallic nanoparticles. 